تأثير الماء المعالج مغناطيسياً على بعض الصفات الفيزيوميكانيكية للبلاستر المحلي(جبس باربس)

$$
\text { عامر عبد الرحيم محمل علي }
$$

تاريخ القبول

ت ت ت اريخ الاستلام

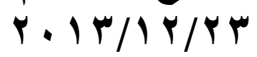

\title{
The Effect of Magnetically Treated Water on some of the Physiomechanical Properties of a Local Plaster (Plaster of Paris)
}

\begin{abstract}
Magnetically treated water was used for the determination of the physiomechanical properties (setting time, water/powder ratio (W/P) and compressive strength) of a local plaster of Paris. The results were compared with that of ordinary, and non-treated water to determine the same properties.

It was found out that through using magnetically treated water, setting time has decreased by an average of $(15.3 \%)$; water powder ratio (W/P) has increased by an average of $(2.4 \%)$ and compressive strength has decreased by an average of $(22.5 \%)$ compared with the results of ordinary water. Therefore magnetically treated water may be considered as an accelerator for the plaster of Paris rather than a retarder.
\end{abstract}

الخلاصة

تم استعمال الماء المعالَج مغناطيسياً لمعرفة تأثيره على بعض الخواص الفيزيوميكانيكية (زمن التجمد ، نسبة الماء إلى المسحوق (W/P) والمقاومة الانضغاطية) على البلاستر المحلي ( بلاستر باريس ) مقارنةً بالماء الاعتيادي غير المعالج لقياس نفس الخواص. 


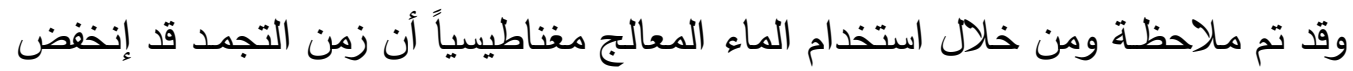

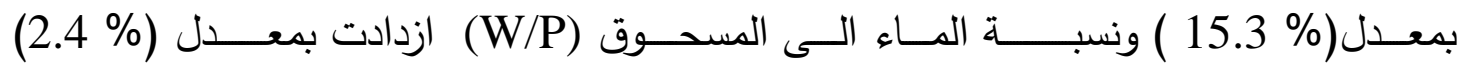

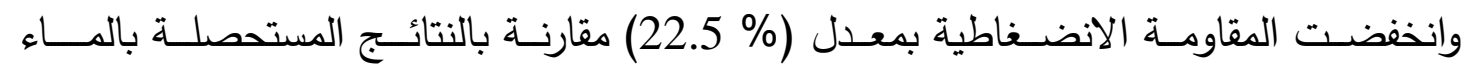
الاعتيـادي. وبنـاءً على ذلك يمكن اعتبار الماء المعالج مغناطيسياً كمادة مسرّعة أو معجّلة (Accelerator) الكلمات الدالة: ماء معالج مغناطيسياً، زمن التجمد ، نسبة الماء الى المسحوق الماند ، المقاومة الانضغاطية ، ماكنيتوترون.

المقدمة الماء الممغنط هو الماء الذي يتم تمريره من خلال مجال مغناطيسي معين بوضع ذلك

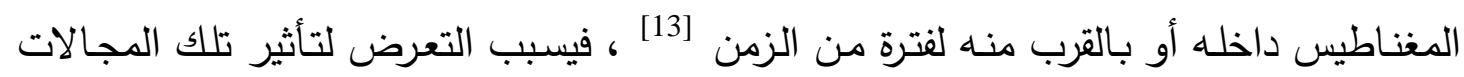

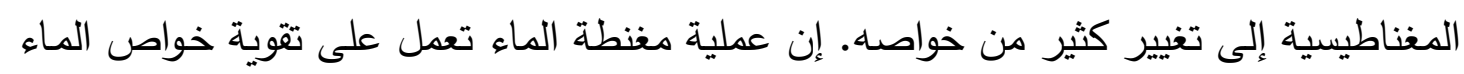
عن طريق تتظيم الثحنات بشكل صحيح (موجب _ سالب موجب ـ سالب), والماء الممغنط يحصل له تغيير في صفاته الكيميائية والفيزيائية المتعددة مثل (زيادة خاصية التوصيل الكهربائي

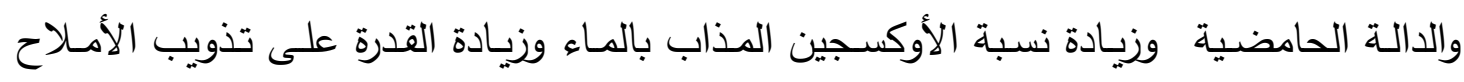
والأحماض و التبلمر والتبلر والتوتر السطحي والتغير في سرعة التفاعلات الكيميائية وخاصية التبخر والتبلل والليونة) [14], [21].

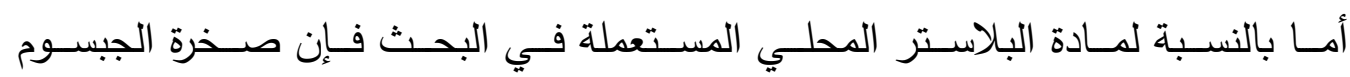
(Gypsum)

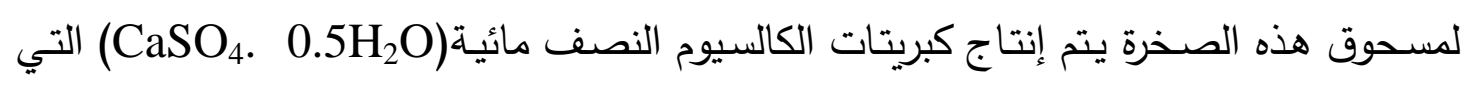

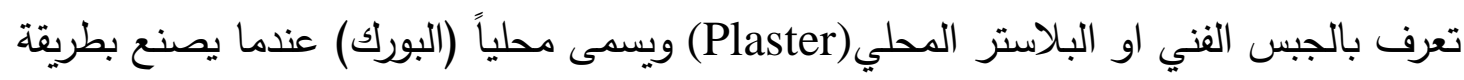

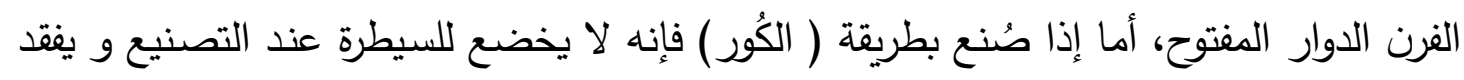

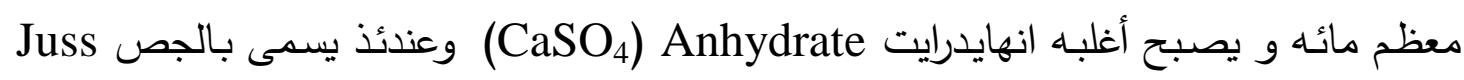

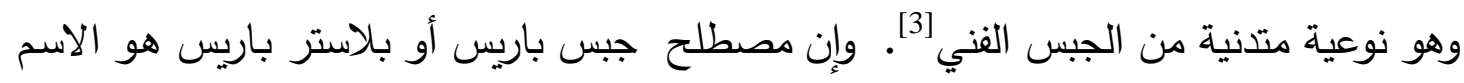

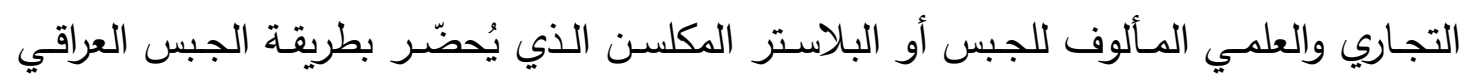

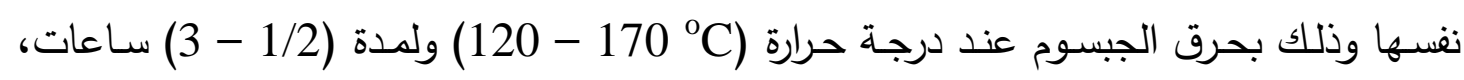

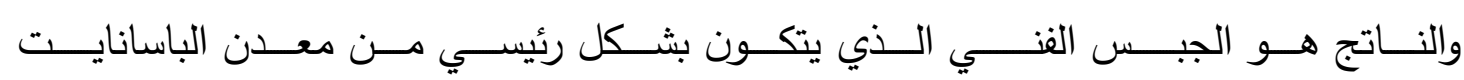

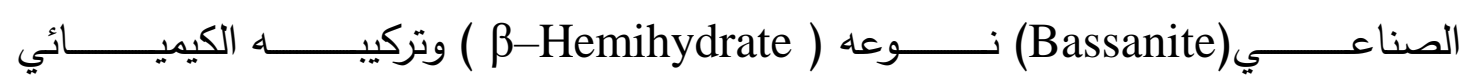

. $\mathrm{CaSO}_{4} \cdot 0.5 \mathrm{H}_{2} \mathrm{O}^{[9]}$ 


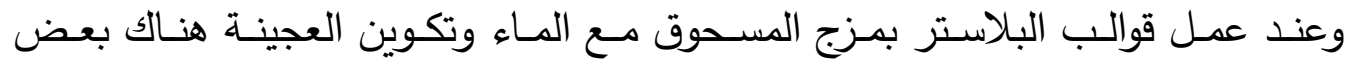

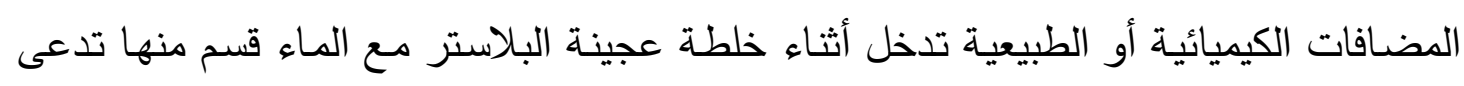

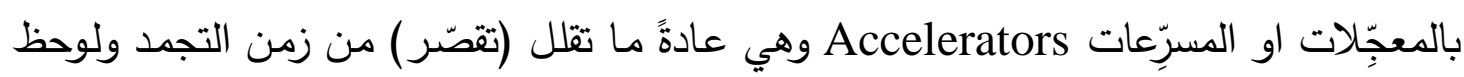

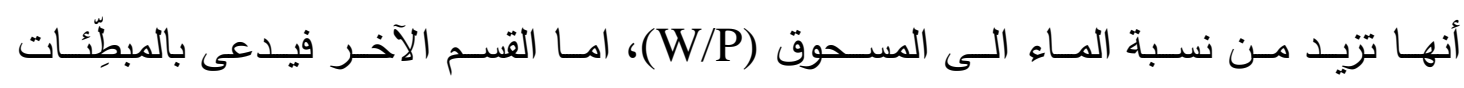
Retarders .[1] (W/P)

أمـا عن أهميـة البلاستر المحلي فهو يـذخل في الكثير مـن الاستخدامات الصـناعية والانشائية والطبية فهو يستعمل في البناء والعزل الحراري (مثل عمل السقوف الثانوية والالواح

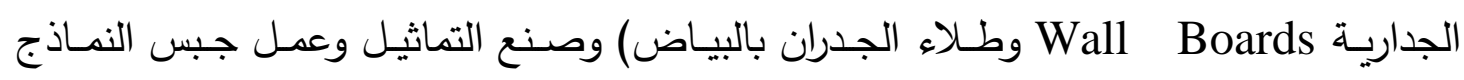
وقوالب الاسنان وتجبير الكسور وغيرها.

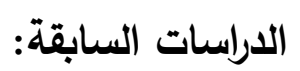

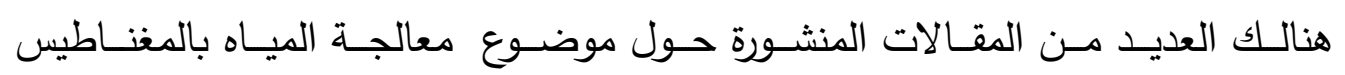
(Magnetic Water Treatment)

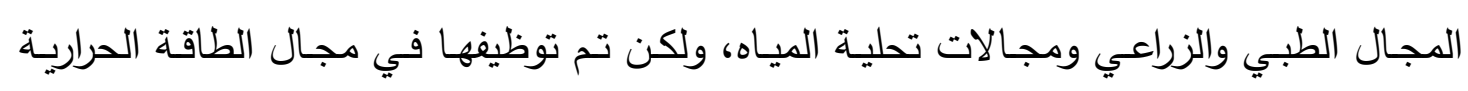

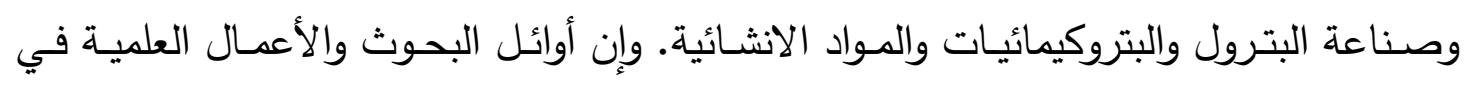

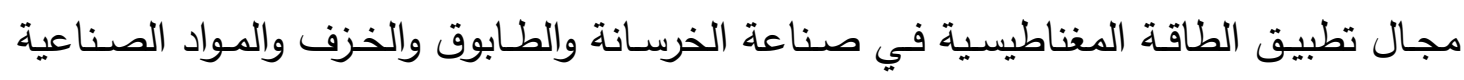
الأخرى بدأت في الاتحاد السوفيتي. ففي البداية كانت هذه الأعمال تجرى في سرية تامة لأنان

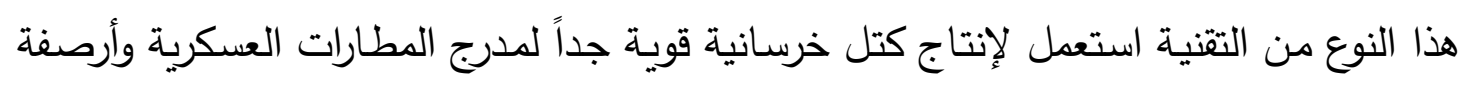

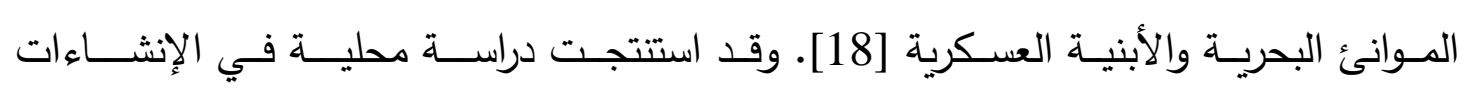

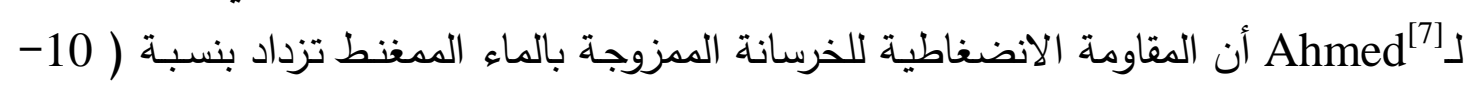
20\%) بالمقارنة مع النماذج المعمولة بالماء الاعتيادي حيث أن استخدام الاسمنت المعد بالمياه

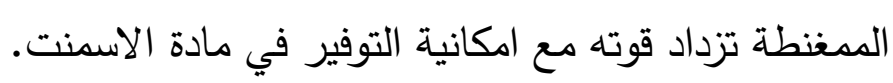

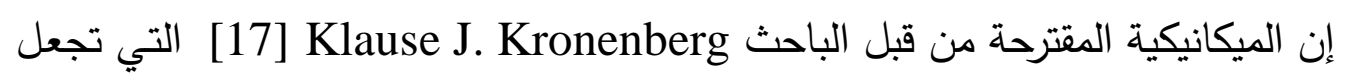

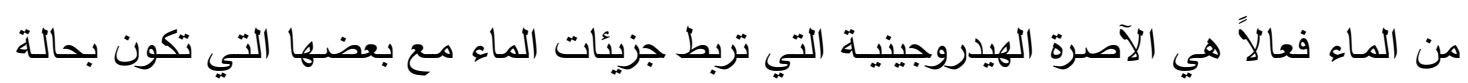

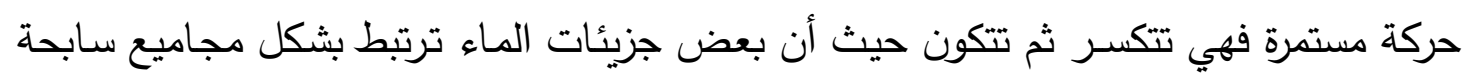

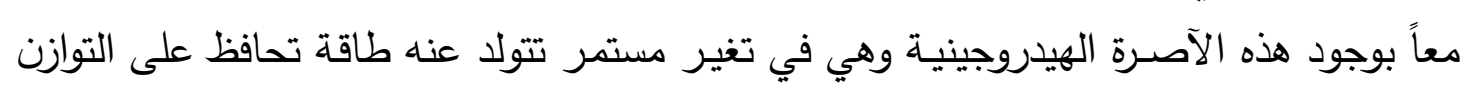
الحركي لجزيئات الماء.

إن المعالجة المغناطيسية للماء لا تمغنط الماء في الحقيقة كما هو شائع خطاً، فالماء ليس كالمواد القابلة للمغنطة عند تعريضها إلى مجال مغناطيسي قوي، لكنه مثل حال السوائل

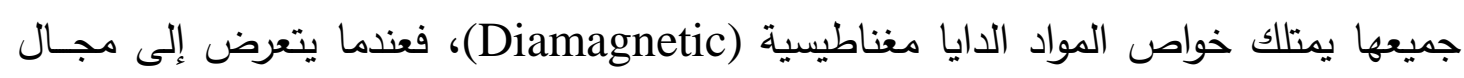


مغناطيسي سوف ينتج الماء مجالاً مغناطيسياً ضعيفاً في الاتجاه المعاكس لذلــك فإن الماء

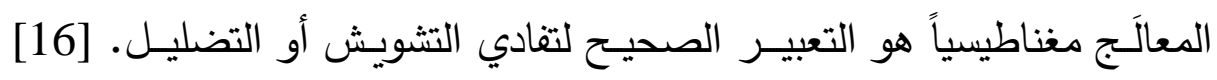

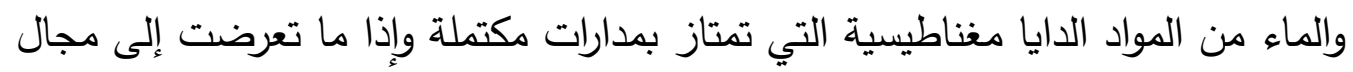

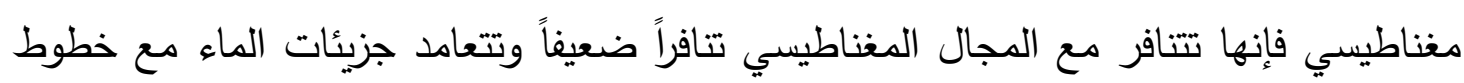

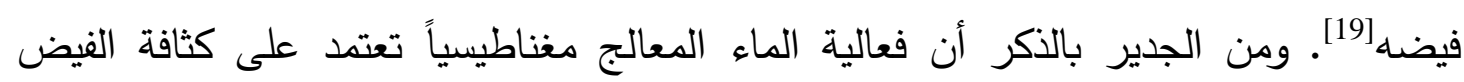

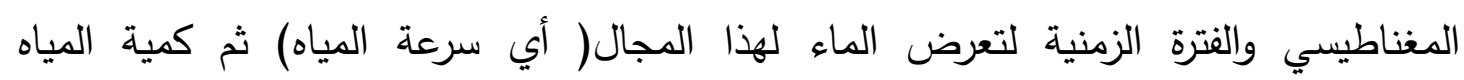
المتعرضة لهذا المجال، وهذه النقاط معتمدة في العديد من بحوث الماء المغناطيسي الحديثة.

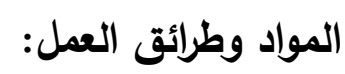
تم شراء (30 kg) من مسحوق بلاستر باريس (Plaster of Paris) من السوق المحلية

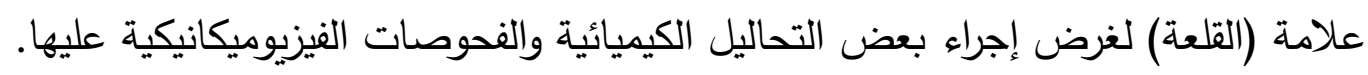
أ- التحاليل الكيميائية: تم إجراء التحاليل الكيميائية والمتضمنة ( I.R. \% , أي الفُضالة غير الذائبة Insoluble Residue) في مختبر الجيوكيمياء / قسم علوم الارض، وكان الهدف من إجراء التحاليل الكيميائية هو حساب كميـة

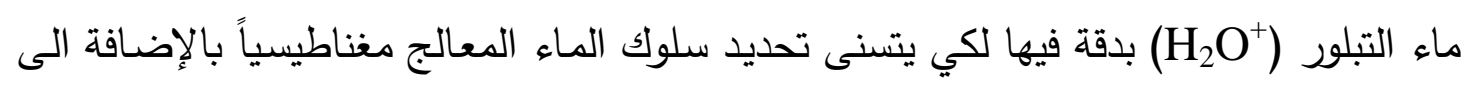

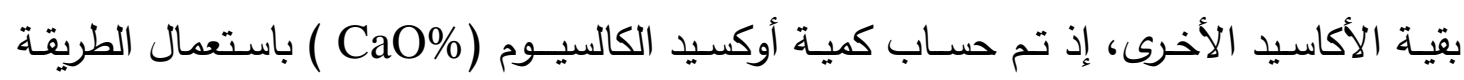

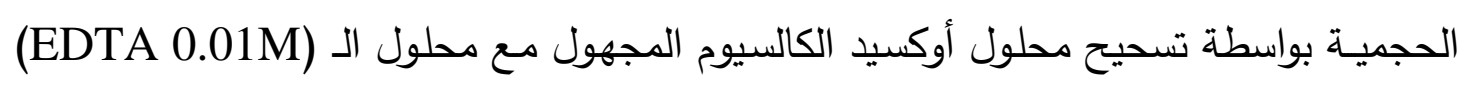

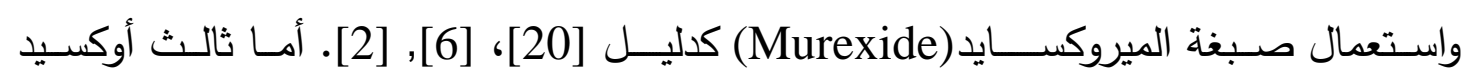

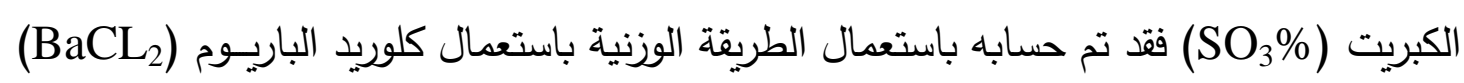

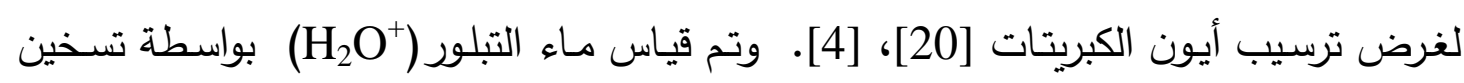
غم من مسحوق النموذج في فرن كهربائي بدرجة حرارة (1gm)

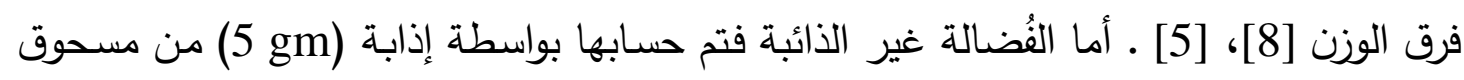

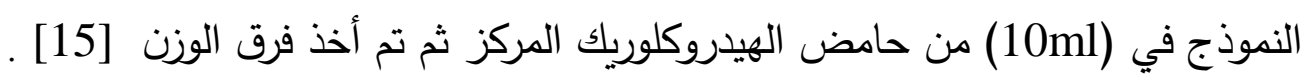

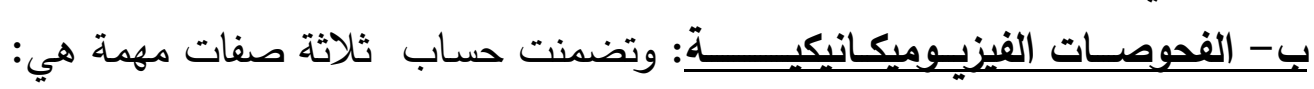

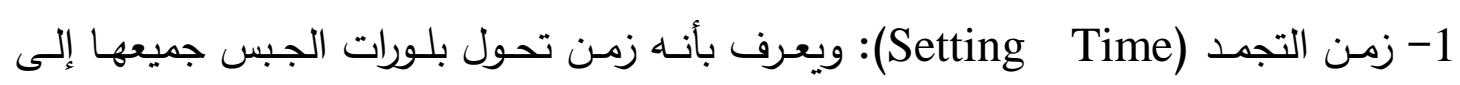

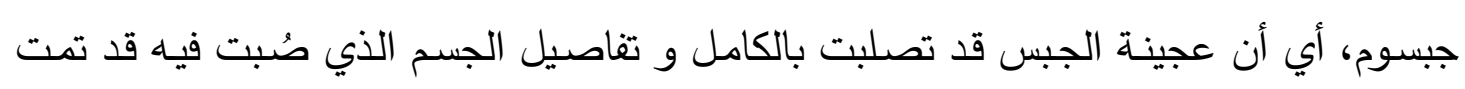

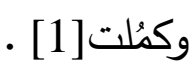

2- نسبـة المـاء إلى المسدــوق (Water - Powder ratio) : بمكن تعريفها بأنها كميـة الدـاء ( مقاسة بال ml) المضافة إلى (Se gm (100) من المسحوق للحصول على عجينة قياسية.

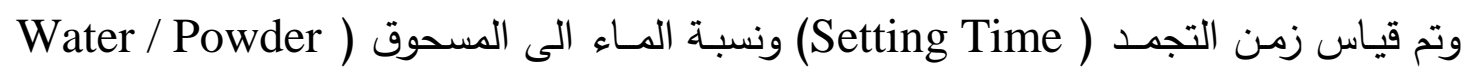

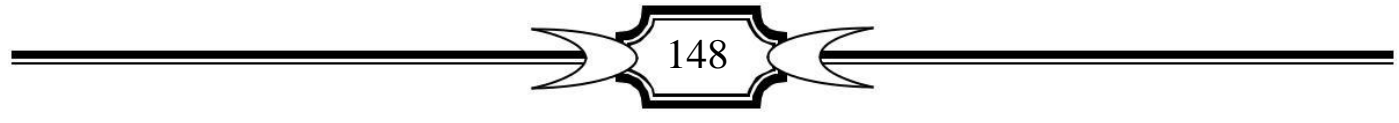


(W/P) (Ratio

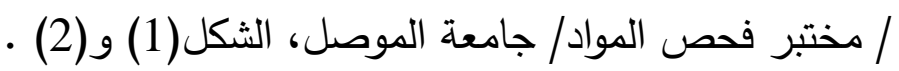

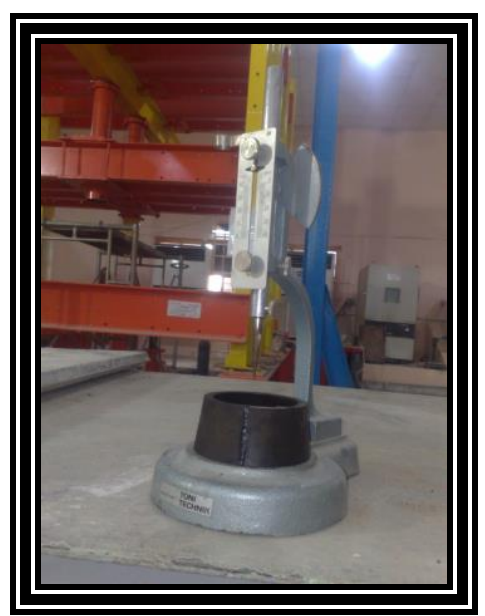

شكل(1) جهاز فيكات لقياس

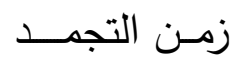

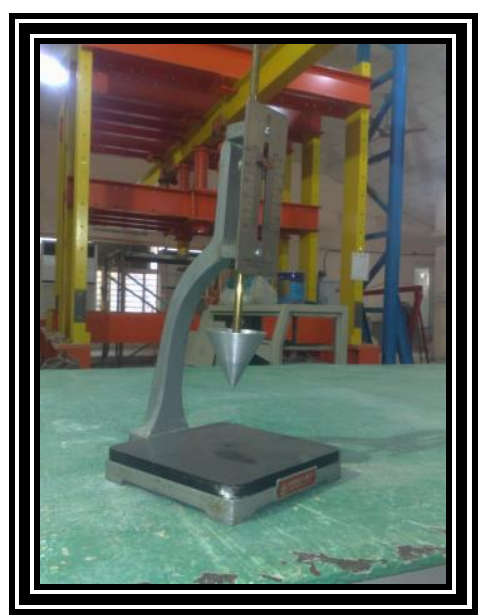

شكل (2) جهاز فيكات لقياس نسبة الماء الى المسحوق (W/P)

3- المقاومـة الانضغاطية Compressive Strength : وهي مدى تحمل النموذج المتصلب

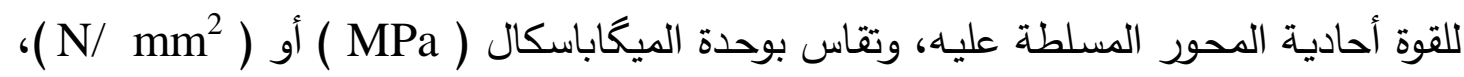

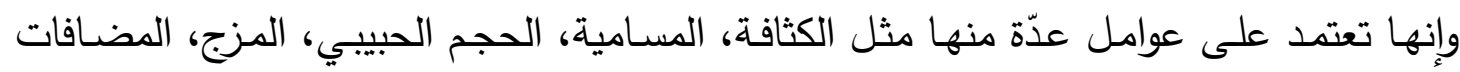

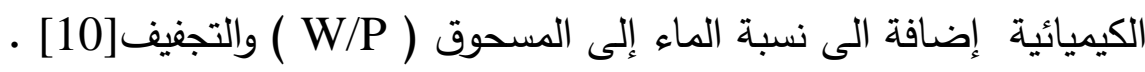

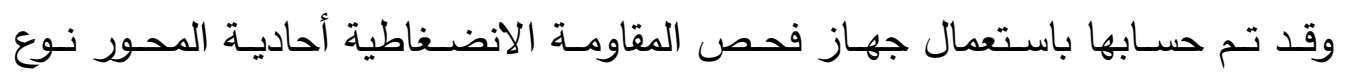

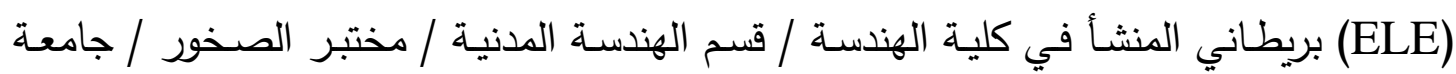
الموصل، والموضح في الثكل (3) بلتئي 


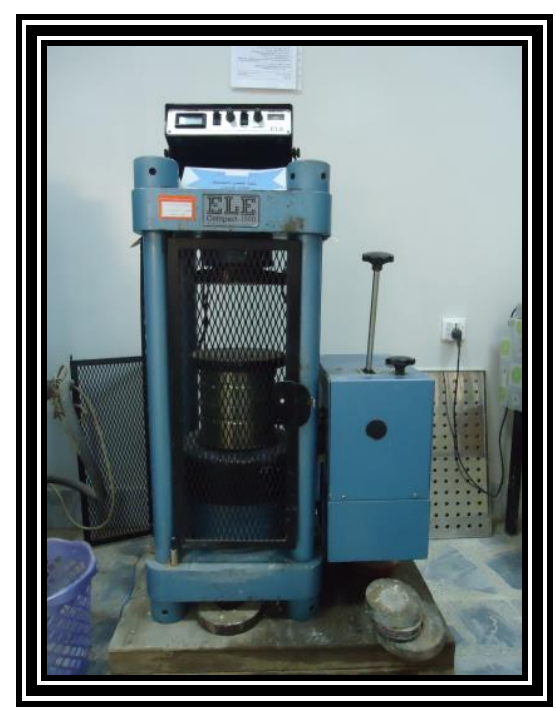

شكل (3) يوضح جهاز قياس المقاومة الانضغاطية نوع ELE

ومن جانب آخر فقد تم معالجة الماء الاعتيادي وتحويله الى ماء معالج مغناطيسياً

باستعمال جهاز (الماكنيتوترون Magnetotron ) بقوة (1800 Gauss) وهو من إنتاج شركة

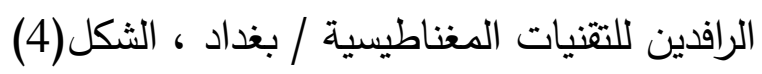

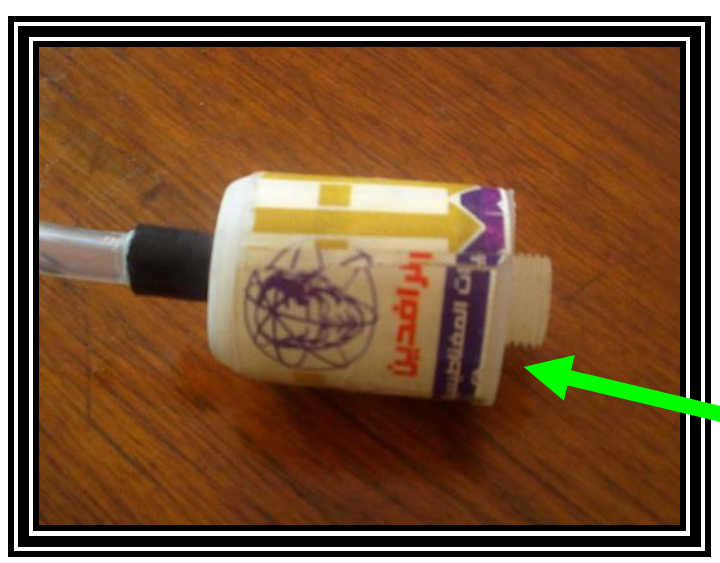

شكل (4-b) الجزء المكبر من جهاز

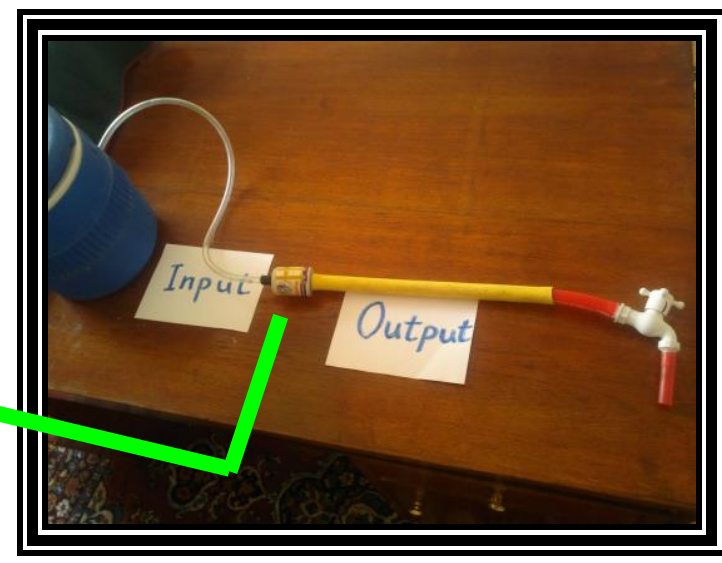

شكل (a) يوضح جهاز الماكنيتوترون

$$
\text { الماكنيتوترون }
$$

وقد تم فحص كثافة فيض المجال المغناطيسي( magnetic flux intensity ) التي

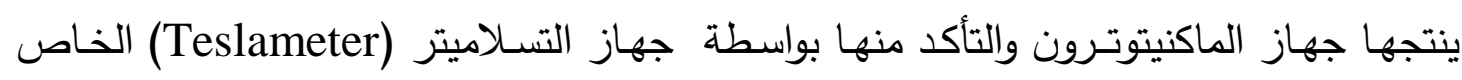
بقياس شدة الفيض المغناطيسي في ( قسم الفيزياء / كلية التربية / جامعة الموصل )، الثكل

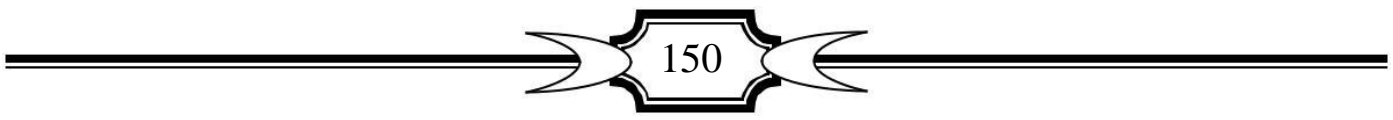




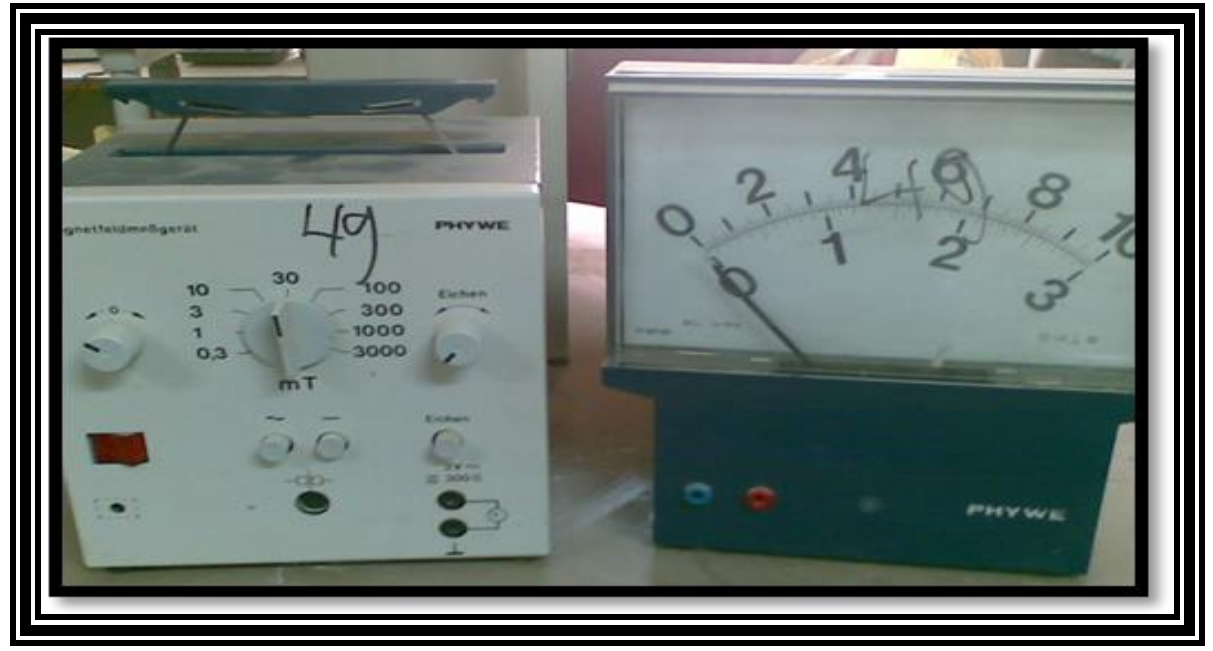

شكل ( 5 ) جهاز التسلاميتز (Teslameter)

\section{النتائج والمناقشة :}

إن نتائج التحاليل الكيميائية لنموذج البلاستر مبينة في الجدول (1). ويوضح الجدولان

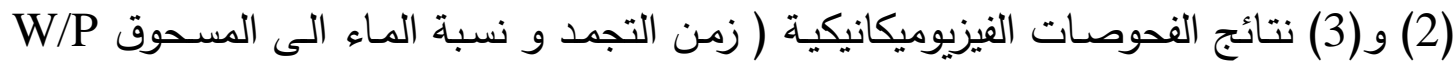
والمقاومة الانضغاطية ) لخمسة حجوم حبيبية مختلفة و التي أجريت على نموذج البلاستر قبل ندئل وبعد استخدام الماء المغناطيسي. إن الهدف من إجراء التحاليل الكيميائية على نموذج بلاستر باريس والتي تم فيها

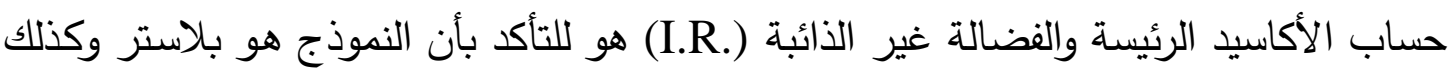
السيطرة على نسبة ماء التبلور ( $\left.\mathrm{H}_{2} \mathrm{O}^{+}\right)$لمعرفة التغيرات الحاصلة في الصفات التهات الفيزيوميكانيكية، ودلّت التحاليل الكيميائية على أن أوكسيد الكالسيوم كمعدل (

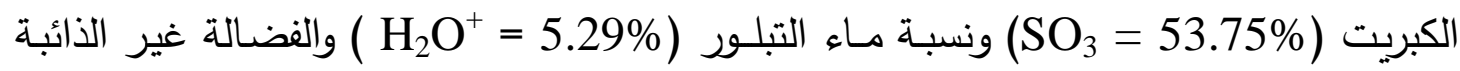

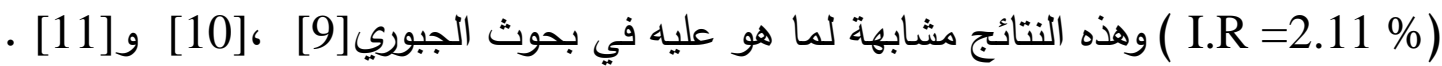

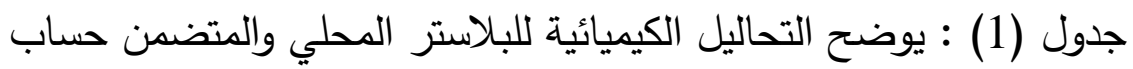
. ( $\mathrm{I} . \mathrm{R} \%, \mathrm{H}_{2} \mathrm{O}^{+} \%, \mathrm{SO}_{3} \%, \mathrm{CaO} \%$ )

\begin{tabular}{|c|c|c|c|c|c|}
\hline Sample & $\mathrm{CaO} \%$ & $\mathrm{SO}_{3} \%$ & $\mathrm{H}_{2} \mathrm{O}^{+} \%$ & $\mathrm{I} . \mathrm{R} \%$ & Total \\
\hline 1 & 37.70 & 53.82 & 5.0 & 1.98 & 98.80 \\
\hline 2 & 38.50 & 53.47 & 5.36 & 2.44 & 99.77 \\
\hline 3 & 38.09 & 53.88 & 5.40 & 2.04 & 99.41 \\
\hline 4 & 37.11 & 53.68 & 5.56 & 1.68 & 99.03 \\
\hline 5 & 37.50 & 53.93 & 5.16 & 2.45 & 98.04 \\
\hline Average & 37.78 & 53.75 & 5.29 & 2.11 & 98.94 \\
\hline
\end{tabular}


أما الفحوصات الفيزيوميكانيكية فقد دلّ الجدولان (2) و(3) على أن هناك تغيراً واضحاً

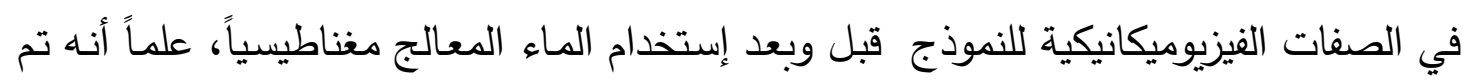
إجراء كافة الفحوصات على نماذج البلاستر المحلي (القلعة) بعد أن تم نخلها باستخدام المناخل ذات الحجوم (Sieves) الحبيبي في بعض الخواص الفيزيوميكانيكية ، إذ أن زيادة الحجم الحبيبي لها تأثيرات عدّة منها

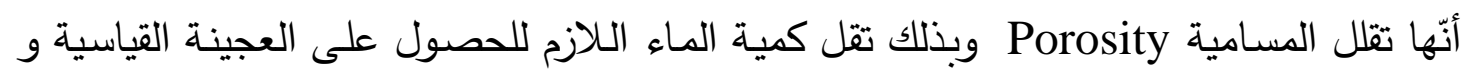
بالتالي زيادة في المقاومة الانضغاطية. ومن خلال نتائج الفحوصـات الفيزيوميكانيكية للبلاستر المحلي قبل وبعد إستخدام الماء

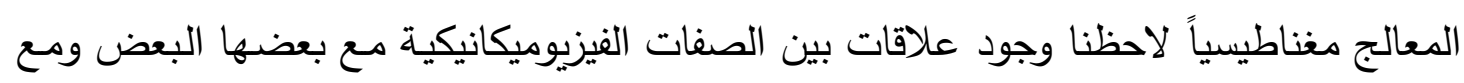
الحجم الحبيبي، يمكن وصفها كما يأتي: أ- وجود علاقة طردية بين الحجم الحبيبي وصفتي زمن التجمد والمقاومة الانضغاطية ، إذ أنه باته

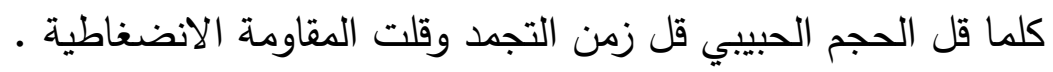
ب- أن العلاقة بين نسبة الماء إلى المسحوق (W/P) و الحجم الحبيبي هي علاقة عكسية

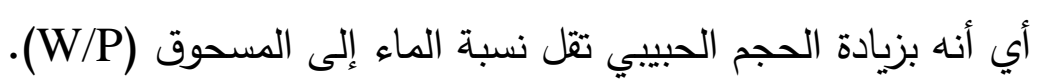

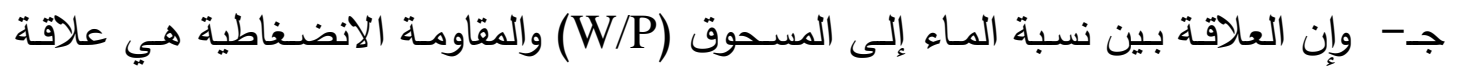

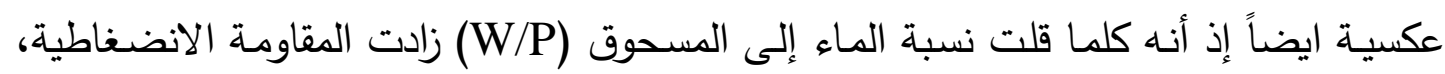

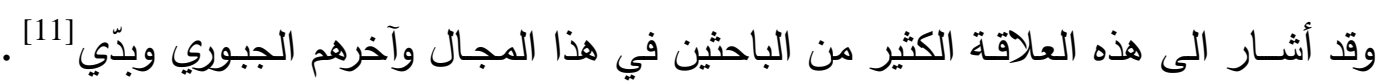

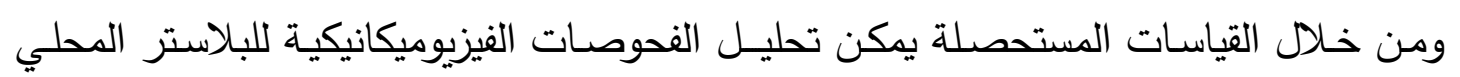

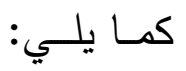
1- زمن التجمد: لوحظ وجود انخفاض(تقليل) في قيم زمن التجمد بإستخدام المـاء المعالج

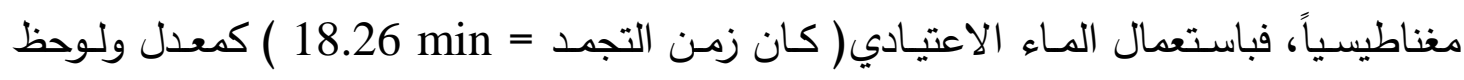

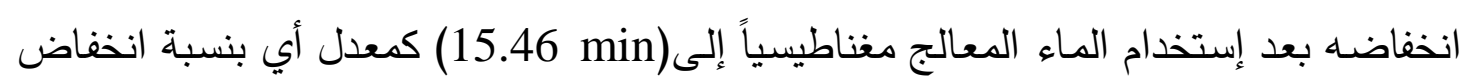
تبلغ (15.3 \%)، وعند حدوث مثل هذا التغير في زمن التجمد فلا بد أن يرافقه تغيرات أخرى في بقية الصفات الفيزيوميكانيكية.

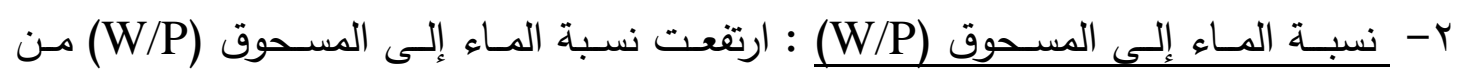

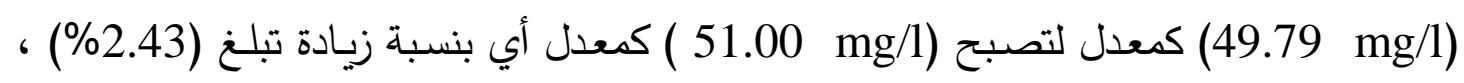

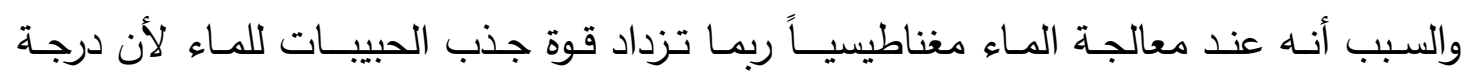

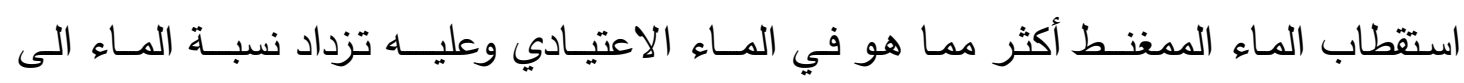


المسحـوق (W/P) للعجينـة القياسيـــة. ويرافق تغير هاتين الصفتين أيضـاً تغير في المقاومـة الانضغاطية.

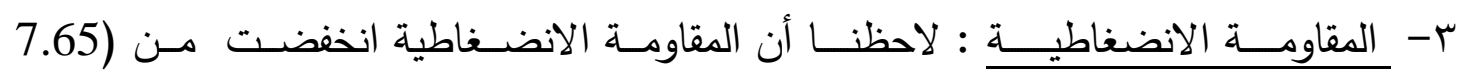

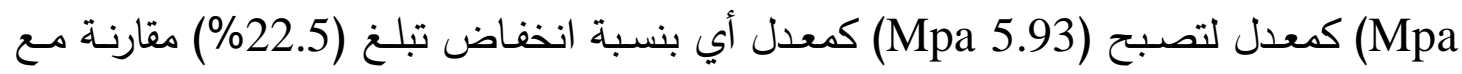

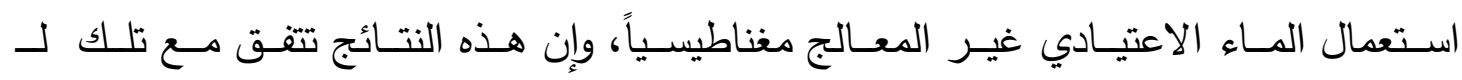
. Bramson[12] جدول (2) : الفحوصات الفيزيوميكانيكية للبلاستر المحلي ( قبل استخدام الماء المعالج مغناطيسياً) والمتضمنة ( (من التجمد , نسبة الماء الى المسحوق , المقاومة الانضغاطية ) لخمسة حجوم حبيبية مختلفة

\begin{tabular}{|c|c|c|c|c|}
\hline $\begin{array}{l}\text { Sample } \\
\text { النموذج }\end{array}$ & $\begin{array}{l}\text { Particle Size } \\
\begin{array}{l}\text { الحجـم الحبيبـي } \\
\text { ( } \mu \mathrm{m})\end{array}\end{array}$ & $\begin{array}{l}\text { Setting } \\
\text { Time } \\
\text { زمن التجمــ } \\
\text { (min.) }\end{array}$ & $\begin{array}{c}\text { W/P } \\
\text { نســبة المـاء إلى المسحوق } \\
\text { (ml/100 gm) }\end{array}$ & $\begin{array}{l}\text { Compressive } \\
\text { strength } \\
\text { المقاومة الانضغاطية } \\
\text { (Mpa) }\end{array}$ \\
\hline 1 & $(250)-(+180)$ & 20.00 & 46.00 & 10.11 \\
\hline 2 & $(180)-(+125)$ & 19.00 & 49.31 & 8.38 \\
\hline 3 & $(125)-(+90)$ & 18.30 & 49.82 & 7.61 \\
\hline 4 & $(90)-(+63)$ & 18.00 & 51.80 & 6.64 \\
\hline 5 & $(63)-(+45)$ & 16.00 & 52.01 & 5.49 \\
\hline Average & & 18.26 & 49.78 & 7.65 \\
\hline
\end{tabular}

جدول (3) : الفحوصات الفيزيوميكانيكية للبلاستر المحلي( باستخدام الماء المعالج مغناطيسياً) والمتضمنة ( زمن التجمد و نسبة الماء الى المسحوق والمقاومة الانضغاطية ) لخمسة حجوم حبيبية مختلفة

\begin{tabular}{|c|c|c|c|c|}
\hline $\begin{array}{l}\text { Sample } \\
\text { النموذج }\end{array}$ & 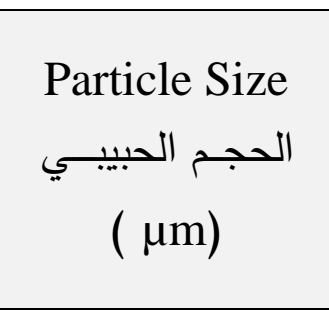 & $\begin{array}{l}\text { Setting } \\
\text { Time } \\
\text { زمن التجمــ } \\
\text { (min.) }\end{array}$ & $\begin{array}{c}\text { W/P } \\
\text { نسـبة المـاء إلى المسحوق } \\
\text { (ml/100 gm) }\end{array}$ & $\begin{array}{l}\text { Compressive } \\
\text { strength } \\
\text { المقاومة الانضغاطية } \\
\text { (Mpa) }\end{array}$ \\
\hline 1 & $(250)-(+180)$ & 17.00 & 48.01 & 7.22 \\
\hline 2 & $(180)-(+125)$ & 16.30 & 50.74 & 6.06 \\
\hline 3 & $(125)-(+90)$ & 16.00 & 50.93 & 5.70 \\
\hline
\end{tabular}




\begin{tabular}{|c|c|c|c|c|}
\hline \hline 4 & $(90)-(+63)$ & 15.00 & 52.60 & 5.48 \\
\hline 5 & $(63)-(+45)$ & 13.00 & 52.74 & 5.19 \\
\hline Average & & 15.46 & 51.00 & 5.93 \\
\hline
\end{tabular}

الاستنتاجات :

1. من خلال إجراء الفحوصات الفيزيوميكانيكية على البلاستر المحلي تبيَّن أن الماء المعالج

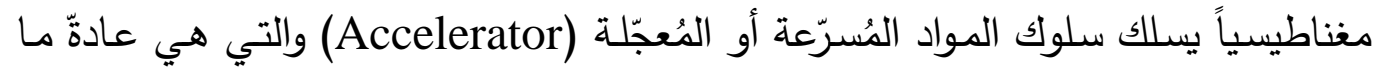
تقلل( تقصّر ) من زمن التجمد وتقلل من قيمة المقاومة الانضغاطية وتزيد من نسبة الماء

$$
\text { الى المسحوق (W/P). }
$$

r. تُشير نتائج الفحوصات الفيزيوميكانيكية أن هناك انخفاضاً واضحاً في زمن التجمد باستخدام

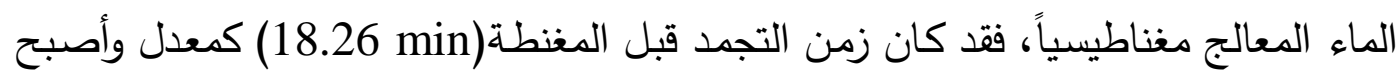

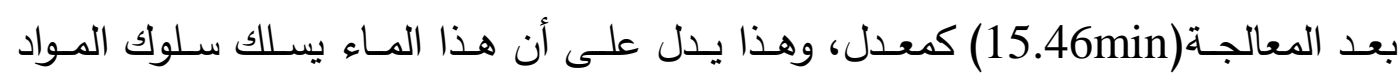
المسرعة. r.نُظهر نتائج الفحوصات الفيزيوميكانيكية إلى أن هناك ارتفاعاً في نسبة الماء الى المسحوق (W/P)

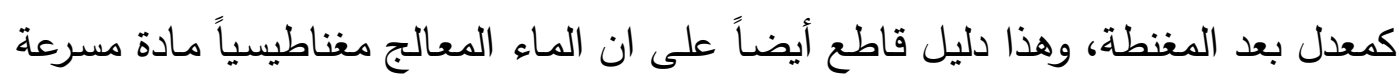
وذلك لأن المواد المسرعة تعمل على زيادة هذه النسبة (W/P).

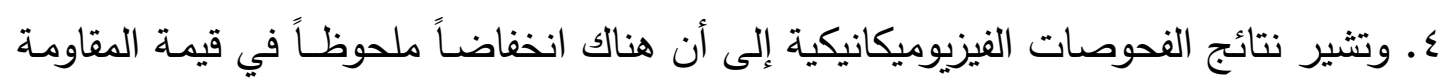

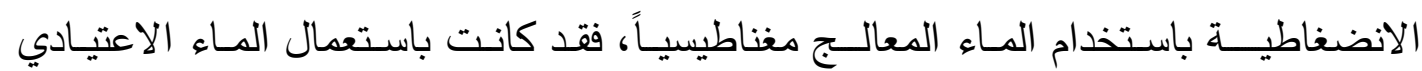

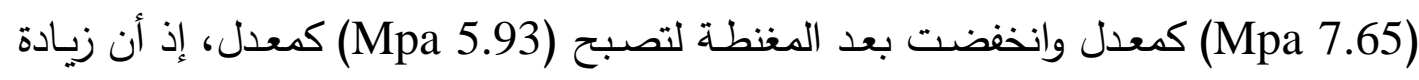
نسبة الماء الى المسحوق (W/P) يؤدي بدوره الى إنخفاض نسبة المقاومة الانضغاطية .

المصادر العربية والانكليزية:

[1] بدي, إبراهيم شيد (2009)" تأثير الانضغاط والمضافات في الصفات الفيزيوميكانيكية للجبس الفني" رسالة ماجستير ، كلية العلوم, جامعة الموصل.

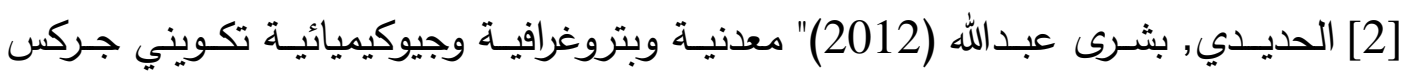

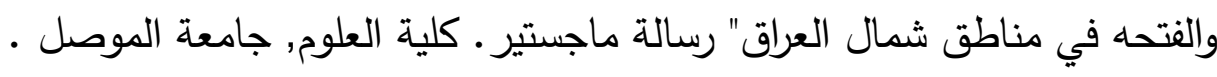

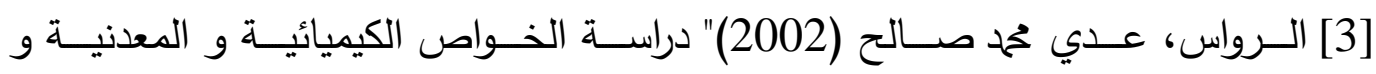

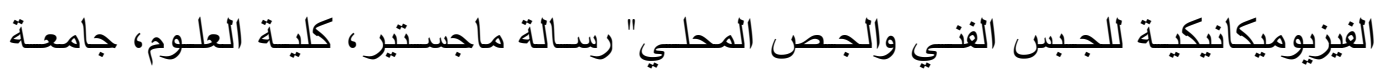
الموصل. 


$$
\begin{aligned}
& \text { [4] سـليمان، مـاهر داود (1990) " جيوكيميائيـة، صـخرية ، أصـل و تحويريـة صـخور } \\
& \text { الجبسوم في تكوين فتحة في منطقة بطمة الغربية - شمال العراق " رسالة ماجستير ، كلية } \\
& \text { العلوم ، جامعة الموصل. } \\
& \text { [5] القره غولي، ناهدة عبد الكريم (1989)" الخواص المعدنية و الكيميائية للجبس العراقي، } \\
& \text { وحدات وطرق التصنيع وتأثيرها على جودة و نوعية الجص و البورلك الناتج" مجلة الجمعية } \\
& \text { الجيولوجية العراقية، مجلد 22، رقم 2، الصفحة(172- 154). } \\
& \text { [6] يوسف, هشام خالد يونس (2011)" بتروغرافية ومعدنية وجيوكيميائية الانواع الثلاثة من } \\
& \text { الجبسوم (العقدي والسلينايت والاليافي)" رسالة ماجستير ـ كلية العلوم, جامعة الموصل. }
\end{aligned}
$$

[7] Ahmed, S. M. (2009) "Effect of Magnetic Water in Engineering properties of Concrete " Al-Rafidain Engineering, Vol. 17, No. 1, pp. 7182.

[8] Aljubouri , Z. A. ( 1972 ) "Geochemistry , Origin and Diagnosis of some Triassic gypsum deposits and associated sediments in East Midland" Ph.D. thesis Univ. of Nottingham, England .

[9] Aljubouri , Z. A. and Al-Rawas, A. M. (2009) "Physical properties and Compressive Strength of the Technical Plaster and Local Juss" Iraqi Journal of Earth Science,Vol. 9, No. 2, pp. 49-58 .

[10] Aljubouri , Z. A. and Baddi I. R. (2012) "Mineralogy, Petrography and Chemistry of two local plasters, Iraq" Iraqi Nat. Jour. of Earth Sci. Vol. 12, No. 2, pp. 1-16

[11] Aljubouri , Z. A. and Dhanoon, A. S. (2012) "The Recycling of Dental Stone from its Hydration product, the gypsum" ( In press).

[12] Bramson, D. (1981) "The Performance of a Magnetic Water Conditioner Under Accelerated Scaling Conditions" Dep. Chem. Eng., Technion Inst. Technol, pp. 217-273.

[13] Dexin, L. et. al., (1992) "Studies of Some Properties of Magnetically Treated Water," Proc. Int. Symp. Phys. Magn. Mater., Vol. 2, pp. 809812.

[14] Donaldson, J. D. (1986) "Magnetic Treatment of Scale Prevention and Descaling in Water Treatment and Process Systems" Prod Finish, Vol. 39, No. 9, pp. 6 -10.

[15] Ellingboe, J. and Wilson, J. A. (1964) "Quantitative separation of non - carbonate minerals from carbonate minerals" Jour. Sed. Petrol Vol. 34, pp.412-418 .

[16] Hoven, V. D. et. al., (1994) "Point-of-Use Treatment: Experience with Physical Water Conditioners in the Netherlands" Kiwa NV, Nieuwegein, Vol. 88, No. 3-8, pp. 281-294.

[17] Kronenberg, K. J. (1985) "Experimental Evidence for Effects of Magnetic Fields on Moving Water" Institute of Electric and Electronic 
Engineers Transactions on Magnetics, Vol. Mag. 21, No. 5, pp. 20592061.

[18] Katz, J. L. (1988) “ Mechanistic Study of the Effects of Magnetic Fields on Scale Formation "Progress Report ,The John Hopkins University.

[19] Sastone, P. and Pandolfo, L. (1994) “Magnetic Treatment of Water and Scaling " Ann. Chim, Vol. 84, No. 5-6, pp. 271-274.

[20] Vogel , I. N. (1961 ) "A Text-book of quantities inorganic Analysis" $3^{\text {rd }}$ edition, Longman, London, England.

[21] Wescott, R. M., (1980) "Nonchemical Water Treating Devices" Materials Performance, pp. 40-42. 